\title{
"Por que escovar os dentes pode fazer bem não apenas à sua saúde, mas também ao seu bolso"†
}

\author{
Cláudio Djissey Shikida* \\ Ari Francisco de Araujo Jr.**
}

RESUMO - Blinder (1974) apresenta o, hoje clássico, modelo de capital humano ampliado para a escovação de dentes. Naquele artigo, entretanto, o autor trata como fixa a renda oriunda de atividades outras que não o trabalho. Neste artigo, uma extensão do modelo é apresentada, seguindo a própria sugestão do autor, através da reinterpretação da renda não oriunda do trabalho como herança. Tal variável dependeria, em parte, da escovação de dentes. Mostra-se que escovar os dentes influencia positivamente no montante herdado, mas ainda assim vale a pena trabalhar.

Palavras-chave: Capital humano. Higiene bucal. Incentivos econômicos.

\section{INTRODUÇÃO}

Em Blinder (1974) apresenta-se um bem-humorado modelo econômico de como a escovação de dentes influencia positivamente na geração de renda (inclusive com evidências econométricas). Entretanto, seu modelo é restrito no sentido de que a renda oriunda de outras fontes que não o trabalho remunerado é exógena. O próprio autor reconhece esta limitação e sugere que o modelo seja ampliado.

Este artigo segue esta sugestão fazendo com que a renda oriunda do não-trabalho (por exemplo, "herança") seja função da escovação de dentes. A dependência funcional da herança com a escovação de dentes pode ser justificada, por exemplo, se imaginarmos que escovar os dentes é uma boa proxy da beleza física que é importante tanto em alguns mercados de trabalho como também em alguns tipos de relacionamentos ${ }^{1}$. Em outras palavras, tratar a

\footnotetext{
† Este texto tem um tom propositalmente irônico, seguindo o estilo de sua principal referência, Blinder (1974). Originalmente, um dos autores teve acesso a este hilariante artigo por meio do prof. João Ricardo Faria (University of Texas at El Paso) ao qual agradecemos (embora, provavelmente, não esperasse que tudo terminasse assim). Também agradecemos ao editor deste periódico que aceitou correr o risco de publicar este artigo, dado o famoso senso de humor dos economistas. Entretanto, acreditamos que o leitor encontrará ao longo do texto várias pistas para desenvolvimento de trabalhos futuros, aplicando o raciocínio econômico a problemas aparentemente não-econômicos (e de maneira mais séria...).

* Doutor em Economia PPGE-UFRGS (Universidade Federal do Rio Grande do Sul). Professor do Ibmec Minas Gerais.

** Mestre em Economia UFMG (Universidade Federal de Minas Gerais). Professor do Ibmec Minas.

${ }^{1} \mathrm{O}$ ex-presidente Fernando Henrique Cardoso submeteu-se a uma cirurgia para retirar suas famosas olheiras e a candidata Dilma Rousseff submeteu-se a uma cirurgia plástica, supostamente, para fins eleitorais. Estes dois exemplos ilustram a importância da beleza no mercado de trabalho, especificamente, no mercado eleitoral. Entretanto, a difusão de métodos de embelezamento artificial nos últimos anos (por exemplo: lipoaspiração,
} 
herança como função da beleza nos ajuda a compreender aspectos da economia do trabalho ${ }^{2}$ e também da economia do casamento e do divórcio ${ }^{3}$ (ou mesmo da economia do crime).

\section{O MODELO}

Sejam $\mathrm{w}=$ salário do indivíduo, $\mathrm{J}=$ índice de trabalho tal que elevados valores de $\mathrm{J}$ correspondem a trabalhos nos quais dentes limpos são importantes, $\mathrm{B}=$ tempo gasto na escovação de dentes, $\mathrm{T}=$ total de tempo, $\mathrm{P}=$ herança. $\mathrm{O}$ objetivo do indivíduo é maximizar sua renda Y, definida como: $Y=w(J, B)(T-B)+P(B)$, onde $w_{B} \geq 0, \quad w_{B J}=w_{J B} \geq 0$ e $P_{B} \geq 0$.

Exatamente como Blinder (1974), supomos w(.) contínua e diferenciável até a segunda ordem, (semiestritamente) quase-côncava e se localizam no quadrante positivo. A novidade é a herança $(\mathrm{P})$, modelada como função do tempo gasto na escovação de dentes (B). Assim, o problema do escovador representativo é:

$$
\underset{B}{\operatorname{Max}} \mathrm{Y}=\mathrm{w}(\mathrm{J}, \mathrm{B})(\mathrm{T}-\mathrm{B})+\mathrm{P}(\mathrm{B})
$$

A condição de primeira ordem é:

$$
\frac{\partial \mathrm{Y}}{\partial \mathrm{B}}=\mathrm{w}_{\mathrm{B}}(\mathrm{J}, \mathrm{B})(\mathrm{T}-\mathrm{B})-\mathrm{w}(\mathrm{J}, \mathrm{B})+\mathrm{P}_{\mathrm{B}}(\mathrm{B})=0 \rightarrow \mathrm{w}_{\mathrm{B}}(\mathrm{J}, \mathrm{B})(\mathrm{T}-\mathrm{B})+\mathrm{P}_{\mathrm{B}}(\mathrm{B})=\mathrm{w}(\mathrm{J}, \mathrm{B})
$$

Rearrumando os termos:

$$
\frac{w_{B}(J, B)}{w(J, B)}(T-B)=1-\frac{P_{B}(B)}{w(J, B)}
$$

Finalmente:

$$
w_{B}(J, B)=\frac{w(J, B)-P_{B}(B)}{(T-B)}
$$

Note que, como $\mathrm{w}_{\mathrm{B}}$, w e $\mathrm{P}_{\mathrm{B}}$ são positivos e como $\mathrm{T}$ - B > 0, então devemos ter: $w(J, B) \geq P_{B}(B)$, o que significa que o salário de equilíbrio é maior do que o acréscimo

botox, silicone, venda de aparelhos de ginástica para usuários domésticos etc.) mostra que o tema é relevante para outros segmentos do mercado de trabalho.

${ }^{2}$ Ver, por exemplo, Hamermesh \& Biddle (1994) e Pfan et al (2000). Mais artigos sobre a relação entre beleza e mercado de trabalho podem ser encontrados <https://webspace.utexas.edu/hamermes/www/Beautystuff.html>.

3 Ver Kuate-Defo (2004) para um estudo não-econômico do mercado de casamentos de homens e mulheres jovens com sugar-mummies e sugar-daddies (algo que poderíamos chamar de "golpe do baú"). 
marginal na herança derivada do tempo gasto na escovação de dentes ${ }^{4}$. Em outras palavras, não vale a pena escovar os dentes pensando primordialmente na herança.

\section{CASO PARTICULAR}

Blinder (1974) considera uma forma funcional particular na qual o salário é linear em B e (potencialmente) não-linear em J. Isto se traduz na equação (4) abaixo. Em nosso caso, seguimos sua sugestão e incluímos uma função linear de $\mathrm{P}$ em B, a equação (5) ${ }^{5}$. Note que, no modelo, assumimos que a herança não é afetada pelo índice J, já que o mesmo diz respeito ao mercado de trabalho. Assim, temos:

$$
\begin{aligned}
\mathrm{w} & =\alpha(\mathrm{J})+\beta(\mathrm{J}) \mathrm{B} \\
P(B) & =\delta+\phi B
\end{aligned}
$$

$\alpha, \beta, \delta, \phi>0$

Derivando (4) e (5) e substituindo em (3), $\operatorname{temos}^{6}$ :

$$
\beta(T-B)=\alpha+\beta B-\phi \rightarrow B=\frac{T}{2}+\frac{\phi-\alpha}{2 \beta}
$$

Note que se $\frac{\phi-\alpha}{2 \beta} \rightarrow 0, B \rightarrow \frac{T}{2}$. Em outras palavras, pessoas cujo salário dependam fortemente de um "sorriso brilhante" (valor elevado de $\beta$ ) gastam metade do seu tempo escovando os dentes ${ }^{7}$. Se, por outro lado, $\beta \rightarrow 0, B \rightarrow 0$, o que significa que empregos nos quais escovar ou não os dentes não faz diferença no salário incentivam o indivíduo a não escovar os dentes ${ }^{8}$.

\section{CONCLUSÃO}

A preocupação com a saúde bucal tem se tornado uma atividade econômica mais lucrativa no Brasil. Segundo Folha (2009), o mercado de antissépticos, creme dental e afins

\footnotetext{
${ }^{4}$ Este é um resultado interessante. Ele implica que se há um bom argumento para convencer as crianças a escovarem os dentes (além da saúde bucal), certamente não é porque, primordialmente, estes receberão uma herança maior. As mães podem respirar aliviadas (e os pais também).

${ }^{5}$ Segue-se exatamente a sugestão do autor, como pode ser confirmado na terceira nota de rodapé do artigo original. É necessário chamar a atenção, contudo, que, neste caso, não existem retornos decrescentes da escovação de dentes sobre a herança recebida ou sobre o salário, i.e., $\mathrm{P}_{\mathrm{BB}}=0 \mathrm{e} \mathrm{W}_{\mathrm{BB}}=0$.

${ }^{6}$ Seguindo Blinder, omite-se os argumentos dos parâmetros variáveis da equação (4).

${ }^{7}$ No apêndice são apresentadas algumas simulações para B, W e P conforme variações em $\beta$, ceteris paribus.

${ }^{8}$ Eis aí uma boa razão normativa para que se incentive profissões "escovação-intensiva", como aquelas nas quais a imagem é importante. Se fizermos a suposição de que o mau hálito possui uma relação monotônica com a escovação no sentido usual, a proposição normativa ganha um apelo mais forte.
} 
mostram uma expansão do setor (“30\% até 2012”) com a generalização do uso desses insumos pela população brasileira. A escovação de dentes provavelmente é uma estratégia de posicionamento do indivíduo no mercado de trabalho. Em outras palavras, além dos apelos tradicionais relacionados à saúde, há um incentivo econômico subjacente à escovação de dentes.

Apesar da escassez de dados sobre a escovação de dentes no Brasil, o que impede a estimação dos impactos do salário sobre o número de escovações de dentes como a feita em Blinder (1974), o modelo simples em questão parece ser promissor no estudo das influências entre um belo sorriso (com dentes limpos), os salários e a herança recebida. Neste sentido, este artigo é uma contribuição teórica aos esforços dos profissionais de saúde que buscam incentivar o maior cuidado com os dentes e com a saúde bucal em geral.

Um resultado interessante diz respeito ao equilíbrio do modelo. De acordo com a condição de primeira ordem, dentes brancos são um incentivo maior para o trabalho, relativamente a atividades aduladoras e relativamente aos potenciais doadores de heranças. Esta é a outra grande contribuição deste artigo, desta vez no campo normativo: escovar os dentes é bom para se obter dinheiro dos outros sem necessidade de inserção no mercado de trabalho, mas é muito mais interessante para você arrumar/manter um emprego. Moralmente falando, é difícil encontrar alguém que queira ensinar o oposto aos seus filhos.

É importante, contudo, notar que o modelo apresentado é de equilíbrio parcial e o mercado de heranças não foi analisado endogenamente. A herança poderia ser endogeneizada como o resultado de adulações" ou de atividades criminosas (por exemplo, o famoso "golpe do baú" $)^{10}$. Além disso, outras hipóteses poderiam ser feitas acerca dos efeitos marginais da escovação de dentes sobre a herança. Estas seriam nossas sugestões para trabalhos futuros.

\section{APÊNDICE}

\subsection{CONDIÇÕES DE SEGUNDA ORDEM}

Dado o problema é:

$$
\underset{B}{\operatorname{aax}} \mathrm{Y}=\mathrm{w}(\mathrm{J}, \mathrm{B})(\mathrm{T}-\mathrm{B})+\mathrm{P}(\mathrm{B})
$$

Condição de primeira ordem:

\footnotetext{
9 Por exemplo, como no famoso Rotten Kid Theorem. Para uma exposição didática do teorema, ver Hirshleifer (1985).

${ }^{10}$ Eventualmente alguém poderia encontrar um resultado interessante: mais escovações de dentes geram menos casamentos por interesses primordialmente econômicos.
} 


$$
\frac{\partial \mathrm{Y}}{\partial \mathrm{B}}=\mathrm{w}_{\mathrm{B}}(\mathrm{J}, \mathrm{B})(\mathrm{T}-\mathrm{B})-\mathrm{w}(\mathrm{J}, \mathrm{B})+\mathrm{P}_{\mathrm{B}}(\mathrm{B})
$$

Condição de segunda ordem:

$$
\frac{\partial^{2} Y}{\partial B^{2}}=w_{B B}(J, B)(T-B)-2 w_{B}(J, B) \leq 0
$$

\subsection{SIMULAÇÕES}

No gráfico 1 abaixo são apresentadas algumas simulações para B, W e P. Note a convergência de $\mathrm{B}$ para $\mathrm{T} / 2$ e o efeito crescente sobre o salário, bem com o efeito decrescente sobre P.

GRÁFICO 1

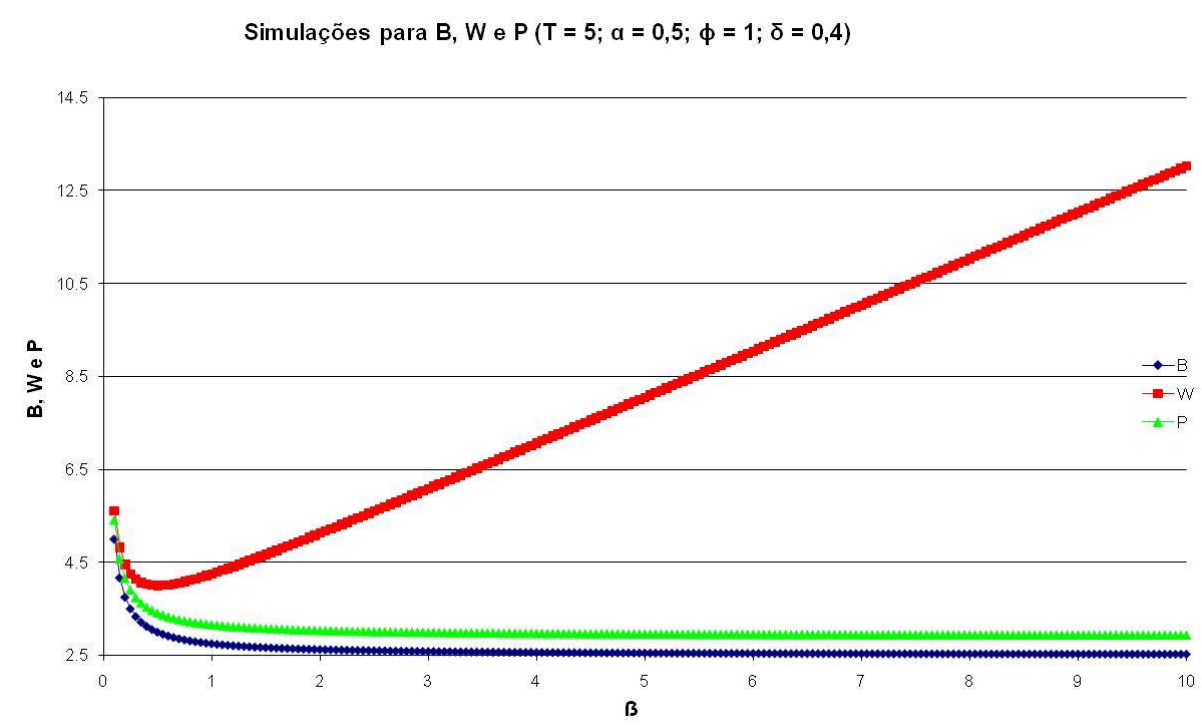

\section{REFERÊNCIAS}

BLINDER, ALAN S. The Economics of Brushing Teeth. Journal of Political Economy, v. 82, n.4. p. 887-91, 1974.

FOLHA DE LONDRINA. O mercado da higiene bucal em expansão. Folha de Londrina, 26 de março de 2009. Disponível em: <http://www.bonde.com.br/bonde.php?id_bonde=139--233-20090326>. Acesso em: 01/4/2010.

HAMERMESH, DANIEL S. \& BIDDLE, JEFF E. Beauty and the Labor Market. American Economic Review, v. 84, n. 5, p. 1174-94, 1994.

HIRSHLEIFER, JACK. The Expanding Domain of Economics. The American Economic Review, v. 75, n. 6, p. 53-68.

KILLINGSWORTH, MARK R. Toward a General Theory of Teeth. The Journal of Human Resources, v. 12, n. 1, p. 125-128, 1977. 
KUATE-DEFO, BARTHELEMY. Young People's Relationships with Sugar Daddies and Sugar Mummies: What do We Know and What Do We Need to Know? African Journal of Reproductive Healty, v. 8, n. 2, p. 13-37, 2004.

PFANN, G.A., BIDDLE, JEFF E., HAMERMESH, DANIEL S. \& BOSMAN, CISKA M. Business Success and Businesses' Beauty Capital. Economic Letters, v. 67, p. 201-7, 2000. 